\title{
Genetic Variation for Field Resistance to Verticillium dahliae Evaluated Using Genotypes and Segregating Progenies of California Strawberries
}

\author{
Douglas V. Shaw \\ Pomology Department, University of California, Davis, CA 95616 \\ W.D. Gubler \\ Plant Pathology Department, University of California, Davis, CA 95616
}

Kirk D. Larson

South Coast Research and Extention Center, Irvine, CA 92718

John Hansen

Pomology Department, University of California, Davis, CA 95616

Additional index words. germplasm, heritability, resistance, fruit breeding

\begin{abstract}
Resistance to wilt caused by Verticillium dahliae Kreb. was evaluated for 41 strawberry genotypes from the Univ. of California breeding program and 1000 offspring from crosses among 23 of these genotypes. Runner plants from these genotypes and seedlings were inoculated with a conidial suspension containing a mixture of five isolates of $V$. dahliae from strawberry. Symptoms were scored as the number of dead or seriously stunted plants per plot, or based on a subjective phenotypic resistance score assigned to each plot on five dates during the spring after planting. Most of the California germplasm is highly susceptible to $V$. dahliae, with an average resistance score of $2.1( \pm 0.10)$ and $84.1 \%( \pm 2.1)$ plants stunted or dead compared with a score of $3.2( \pm 0.24)$ and $57.4 \%( \pm 4.9)$ of plants stunted or dead for a control set of six nonCalifornia genotypes identified previously as resistant. However, a broad range of intermediate resistance was detected, and 4 of the 41 California genotypes evaluated had resistance scores superior to the mean score for the non-California resistant checks. Plot-mean heritabilities for resistance and stunting scores estimated using genotypic, full-sib family, and offspring-parent analyses ranged from 0.44 to 0.88 . Comparison of different estimates of variance components suggests that half or more of the genotypic variance for resistance traits detected is due to the additive effects of genes. There appears to be sufficient variation within the California population to proceed with an effective selection program, despite the absence of directional selection for resistance during the past 3 decades. However, developing cultivars with adequate resistance will ultimately depend on the recovery of transgressive segregants from superior parents, as even the most resistant genotypes from all sources showed some disease symptoms.
\end{abstract}

Early this century and through the 1970s, Verticillium dahliae was considered among the most important pathogens limiting strawberry production in California (Thomas, 1932). Verticillium wilt continues to be a major concern where perennial strawberry plantings are used (Maas et al., 1989; Paulus, 1990). Together, successful preplant soil fumigation methods (Wilhelm and Koch, 1956) and annual planting systems (Voth and Bringhurst, 1990), both of which were widely adopted in the 1970s, virtually eliminated severe Verticillium soil infestations of and obviated the need for resistant cultivars (Wilhelm et al., 1961; Wilhelm and Paulus, 1980). Restrictions that may limit the use of the most effective soil fumigants have generated new interest in genetic resistance.

Before the widespread use of soil fumigants, genetic solutions were considered one option for mitigating the Verticillium problem in California, and breeding for resistance or tolerance received extensive effort. Wild germplasm was found to harbor extensive variation, although none of the genotypes evaluated in those studies had resistance levels sufficient to withstand high inoculum densities (Bringhurst et al., 1966). Extensive testing of commercial strawberry breeding populations detected relatively high heritabilities for resistance based on plot-mean evaluations and suggested that resistance is conditioned by additive and dominance

Received for publication 21 Nov. 1995. Accepted for publication 12 Feb. 1996. The cost of publishing this paper was defrayed in part by the payment of page charges. Under postal regulations, this paper therefore must be hereby marked advertisement solely to indicate this fact. genetic effects (Bringhurst et al., 1968). Small negative genetic correlations were detected between resistance and productivity, whereas small positive correlations were detected between resistance and fruit firmness (Bringhurst et al., 1968). Productivity and fruit firmness have increased in the California breeding population during the past 3 decades, and the consequences of combined unconscious selection and genetic sampling on genetic variation for resistance to $V$. dahliae are difficult to predict.

The California breeding population has been essentially closed during the past 30 years, with little or no infusion of germplasm (Shaw et al., 1989). Any variation for resistance detected in the current population has survived despite an absence of direct efforts to improve genetic resistance. The purpose of the experiments described below was to estimate the magnitude and determine the distribution of genetic variance for field resistance to wilt caused by $V$. dahliae in a population of advanced selections that could serve as parent genetic stock in the Univ. of California strawberry improvement program. Results form these experiments should permit an evaluation of the need for germplasm infusion and an indirect assessment of the empirical consequences of any negative genetic correlations between resistance and productivity.

\section{Materials and Methods}

Forty-one genotypes from the California breeding population and six cultivars identified as resistant (Maas, 1984; Maas et al., 1989) were evaluated using replicated plots of runner plants. The 
California germplasm included 14 cultivars ('Anaheim', 'Camarosa', 'Carlsbad', 'Chandler', 'Cuesta', 'Irvine', 'Laguna', 'Lassen', 'Parker', 'Pajaro', 'Seascape', 'Selva', 'Sunset', and 'Yolo') and 27 advanced selections. The six non-California check cultivars were 'Earliglow', 'Guardian', 'Lateglow', 'Ranier', 'Tribute', and 'Tristar'. It is important to note that complete resistance to $V$. dahliae in domestic strawberry germplasm is extremely rare. For example, Maas et al. (1989) scored only one Fragaria ×ananassa genotype and two $F$. chiloensis genotypes as exceptionally resistant, with all others tested showing some disease symptoms. The check cultivars used here exhibit partial resistance, and are representative of the most resistant strawberry cultivars documented to date.

Verticillium dahliae isolates were obtained from symptomatic strawberry plants growing near Watsonville, Calif. Isolates were single spored and maintained on potato dextrose agar (PDA) slants at $7^{\circ} \mathrm{C}$. For inoculum preparation, isolates were grown on acidified PDA for 14 days, after which conidia were removed by flooding plates with sterile distilled water and scraping the mycelium with a sterile glass rod. Water containing the conidia was strained through four layers of cheesecloth to filter out mycelial fragments. Final concentration was adjusted to $10^{6}$ conidia/ml after mixing five isolates as recommended by Bringhurst et al. (1961).

Field trials were established at the Wolfskill Experimental Orchard near Davis, in central California. The experimental site was prepared for planting and fumigated with 67 methyl bromide : 33 chloropicrin at $392 \mathrm{~kg} / \mathrm{ha}$. Runner plants from each genotype were harvested from an adjacent nursery on $27 \mathrm{Sept}$. 1993, trimmed to retain two leaves, and stored at $2{ }^{\circ} \mathrm{C}$ until planting on $30 \mathrm{Sept}$. (Shaw, 1993). Before planting, all plants were dipped for $5 \mathrm{~min}$ in the inoculum solution containing about $10^{6}$ conidia $/ \mathrm{cm}^{3}$. Thereafter, plants were treated according to recommendations for commercial winter plantings (Welch, 1989). Each genotype was represented in the test by two plots of five runner plants, one randomized within each of two complete blocks.

In addition, seedlings from 50 biparental progenies, generated by crosses among 23 of the California cultivars and advanced selections (with one to six crosses per parent), were inoculated and evaluated for resistance to wilt. Germination began on 17 June 1993; germinants were transplanted to peat pots during the first week of August, then inoculated and transplanted to the field on 30 Sept. Seedlings were inoculated using the same conidial suspension as for runner plants. To inoculate seedlings, water was withheld from individual peat cells, then the desiccated cells were placed in a shallow pan (1 cm deep) containing $1 \mathrm{~L}$ of the suspension. Seedlings were transplanted to the field after absorbing about $15 \mathrm{~mL}$ of the suspension. Each progeny was represented in the test by two plots of ten seedlings, with one plot randomized within in each of two blocks.

Symptoms were scored as the number of dead or seriously stunted plants per 5- or 10-plant plot and, based on a subjective phenotypic resistance score assigned to each plot. The subjective resistance score was rated on a scale from 1 to 5 , where $1=$ severe verticillium wilt symptoms (in most cases, complete mortality) and $5=$ no detectible disease symptoms. Plots of runner plants were evaluated on six dates during the spring after planting: $28 \mathrm{Apr} ., 21$ May, 27 May, 9 June, 10 June, and 27 June. Plots of seedlings were evaluated on all of the above dates except 21 May. Altogether, four different observers performed either one or two of the six evaluations. The presence of $V$. dahliae in symptomatic plants was verified by sampling leaves from 54 randomly chosen plants with symptomatic leaves. Petioles were surface-sterilized for $30 \mathrm{~s}$ in $0.5 \%$ sodium hypochlorite, after which cross-sections were placed on water-agar and plates were read 10 days after plating. Positive $V$. dahliae isolations were obtained from all symptomatic plants.

Further statistical analyses were conducted using the average values for each variable over all six observation dates. The numbers of stunted or dead plants were converted to percentages to normalize the scales for runner and seedling experiments, and the percentages were then treated with an arcsine-square root transformation before statistical analyses.

Analyses of variance (ANOVAs) were conducted with blocks treated as fixed effects, and either genotypes or crosses as random effects, using SAS's GLM procedure and Type III sums of squares (SAS, 1988). Tests of significance were conducted and variance components were estimated using linear functions of the expected mean squares obtained through SAS analyses. The genetic components of variance inferred from genotypic and biparental cross analyses, $\sigma^{2}$ and $\sigma^{2}$, respectively, have different genetic expectations and interpretations: $\sigma^{2}$ reflects the total complement of genetic variance components, whereas the genetic expectation of $\sigma^{2}$ for biparental crosses is $\sigma_{c}^{2}=0.5 \sigma^{2}{ }^{2}+0.25 \sigma^{2}{ }_{d}$, plus small fractions of epistatic components (Comstock and Robinson, 1948).

Plot-mean heritabilities were estimated on a genotypic basis $\left(\mathrm{H}_{\mathrm{p}}^{2}\right)$ as (Shaw, 1993)

$$
\mathrm{H}_{\mathrm{p}}^{2}=\sigma_{\mathrm{g}}^{2} /\left(\sigma_{\mathrm{g}}^{2}+\sigma^{2} / \mathrm{r}\right)
$$

and seedling plot mean heritabilities $\left(\mathrm{h}_{\mathrm{p}}^{2}\right)$ were estimated as

$$
\mathrm{h}_{\mathrm{p}}^{2}=\sigma_{\mathrm{c}}^{2} /\left(\sigma_{\mathrm{c}}^{2}+\sigma^{2} / \mathrm{r}\right)
$$

In Eq. [1], $\sigma^{2}$ includes only among-plot error, whereas this parameter in Eq. [2] includes the consequences of measurement error and genetic sampling. In both equations, $r$ is the number of experimental plots.

A third heritability estimate $\left(\mathrm{h}_{\mathrm{pr}}^{2}\right)$ was obtained by regression of offspring plot values on the corresponding midparental means. An estimate of the additive genetic variance $\left(\sigma_{\mathrm{a}}^{2}\right)$ was also obtained from offspring-parent comparisons as $2\left(\mathrm{COV}_{\mathrm{OP}}\right)$, where $\mathrm{COV}_{\mathrm{OP}}$ is the covariance of offspring and midparental values (Falconer, 1981), obtained using SAS's CORR procedure.

All of the above heritabilities were calculated on a plot-mean basis, and their values will differ when evaluations are based on plots of different sizes. Inference about selection response must be limited to experiments with similar design, especially with differing numbers of plots per entry or plants per plot. Specifically, $\mathrm{H}^{2}$ is appropriate to predicting genotypic selection response, $\mathrm{h}^{2}$ would be useful to predict response for selection of superior crosses, and $\mathrm{h}_{\mathrm{pr}}^{2}$ would predict the cumulative response expected in the offspring of selected genotypes.

Because expected values for genetic parameter estimates do not depend on the experimental design, broader inference about the inheritance of these indicators of resistance can be obtained by pairwise comparison of the estimated genetic components of variance and covariance from genotypic, cross, and regression sources. Equating pairs of experimental genetic components of variance and covariance to their model expectations provides three estimates of the additive and dominance genetic variance. These comparisons are confounded in that the three studies may reflect differences in inoculation procedure between seedlings and runners, and perhaps differences in resistance associated with differing root physiology or growth patterns between these propagule types. As a consequence, the distribution of genetic variance among additive and dominance sources must be interpreted with some caution. However, other methods for direct extraction of 
genetic variance components (see Nelson et al., 1995) are expected to give unreliable results when the number of crosses per parent is small and variable, as in our seedling population; likewise, generalized progeny analyses ignore the information available through simultaneous evaluation of clonal and seedling trials.

To assess the relative importance of disease incidence and the subjective symptom scores, genetic correlations between these traits were calculated on a genotypic or cross basis following Shaw (1993).

\section{Results and Discussion}

Most of the California germplasm evaluated was highly susceptible to $V$. dahliae. California genotypes had an average resistance score of $2.1( \pm 0.10)$ and stunting or mortality of $84.1 \%( \pm 2.1)$, compared with values of $3.2( \pm 0.24)$ and $57.4 \%( \pm 4.9)$ for the resistant control set of six non-California genotypes (Table 1). The range of resistance scores observed was substantial, within both the California genotypes and the resistant control set (Table 1), and all genotypes exhibited stunting to some extent. However, except for 'Lateglow' (score $=1.80 \pm 0.40$ ), all of the non-California checks had resistance scores $>3.30$. Of the 41 California genotypes evaluated, 4 had resistance scores superior to the mean scores for the non-California resistant checks; two of these are cultivars: 'Yolo' (score $=3.60 \pm 0.00$ ) and 'Laguna' (score $=4.50 \pm 0.10$ ). Resistance scores for cultivars currently important to strawberry production in California ('Camarosa', 'Chandler', 'Pajaro', 'Seascape', 'Selva') ranged from 1.00 to 2.40 , and none of these demonstrated exceptional levels of resistance to verticillium wilt. On average, seedlings were less affected than runner plants (Table $1)$, with a resistance score of $2.8( \pm 0.068)$ and stunting or mortality of $60.7 \%( \pm 2.0)$.

Highly significant genetic variances were detected in genotypic and progeny analyses (Tables 2 and 3). Genotypic and cross-mean heritabilities were large $\left(\mathrm{H}^{2}{ }_{\mathrm{p}}=0.84-0.88, \mathrm{~h}_{\mathrm{p}}^{2}=0.61-0.88\right)$, suggesting that selection for either superior genotypes or crosses that exhibit a high level of resistance should be effective, at least in the short term. Heritability was smallest for percent stunting or mortality evaluated for biparental cross means $\left(\mathrm{h}_{\mathrm{p}}^{2}=0.61\right)$, possibly the result of an inoculation procedure that is less reliable than that for runner plants and that permits a higher frequency of escapes. Genetic correlations between percent stunting or mortality and subjective scores were -0.958 and -0.933 for calculations based on crosses and genotypes, respectively. This result indicates that both variables reflect a nearly identical genetic mechanism for resistance and further supports the contention that heritability for percent stunting or mortality in seedlings is lower than for genotypes as a consequence of inoculation heterogeneity.

Highly significant regressions were also detected for both traits (Table 4). Offspring-parent regression heritabilities were somewhat smaller than those calculated for crosses and genotypes $\left(\mathrm{h}_{\mathrm{pr}}^{2}\right.$ $=0.44-0.47)$. The variance components that reflect genetic differences among crosses and genotypes each contain causal components due to dominance and epistasis, whereas the covariance of offspring and parent depends only on additive effects and fractional additive $\times$ additive epistatic components. Thus, larger heritability estimates based on the variances of crosses and genotypes suggest the presence of some type of nonadditive genetic variation for resistance to $V$. dahliae. This comparison is weakened by the fact that the inoculation procedures and the physiological status of the plants differed for crosses and genotypes, which might cause relative resistance to be conditioned by differing sets of genes in each case.
Parameter estimates for additive and dominance genetic variance obtained from comparison of genotypic, cross, and offspring-parent analyses (Table 5) generally show additive genetic variance to be equal to or somewhat larger than dominance variance. The only case for large dominance variance derives from comparing genotypes and crosses with respect to the percent stunted or dead plants $\left(\sigma_{\mathrm{a}}^{2} / \sigma_{\mathrm{d}}^{2}=0.55\right)$. The remaining ratios are

Table 1. Means, standard errors (in parentheses), and ranges (in brackets) for variables scored to describe resistance to Verticillium dahliae in three strawberry populations.

\begin{tabular}{lccc}
\hline \hline & $\mathrm{N}$ & $\begin{array}{c}\text { Stunted or dead } \\
(\%)\end{array}$ & $\begin{array}{c}\text { Resistance } \\
\text { score }\end{array}$ \\
\hline Genotype & 82 & 84.1 & 2.12 \\
& & $(2.1)$ & $(0.10)$ \\
& & {$[22 \%-100 \%]$} & {$[1.00-4.5]$} \\
Resistant genotype & 12 & 57.4 & 3.23 \\
& & $(4.9)$ & $(0.23)$ \\
Seedling & & {$[34 \%-88 \%]$} & {$[1.8-3.9]$} \\
& 100 & 60.7 & 2.80 \\
& & $(2.0)$ & $(0.07)$ \\
& & {$[21 \%-96 \%]$} & {$[1.5-4.5]$} \\
\hline
\end{tabular}

Table 2. Analysis of variance results and plot-mean heritability estimates for variables scored to describe resistance to Verticillium dahliae in California strawberry genotypes.

\begin{tabular}{lrccc}
\hline \hline Source & df & $\begin{array}{c}\text { Expected } \\
\text { mean squares }\end{array}$ & $\begin{array}{c}\text { Stunted or dead } \\
(\%)\end{array}$ & $\begin{array}{c}\text { Resistance } \\
\text { score }\end{array}$ \\
\hline Block & 1 & $\sigma^{2}+82 \sigma_{\mathrm{b}}^{2}$ & 0.001 & $0.871^{*}$ \\
Genotype & 40 & $\sigma^{2}+2 \sigma_{\mathrm{g}}^{2}$ & $0.148^{* *}$ & $1.428^{* *}$ \\
Error & 40 & $\sigma^{2}$ & 0.023 & 0.172 \\
$\sigma^{2}$ & & & 0.062 & 0.624 \\
$\mathrm{H}^{2}$ & & & 0.84 & 0.88 \\
$\mathrm{SE}$ & & & 0.22 & 0.22 \\
\hline
\end{tabular}

Table 3. Analysis of variance results and cross-mean heritability estimates for variables scored to describe resistance to Verticillium dahliae in California strawberry progenies.

\begin{tabular}{lrccc}
\hline \hline Source & df & $\begin{array}{c}\text { Expected } \\
\text { mean squares }\end{array}$ & $\begin{array}{c}\text { Stunted or dead } \\
(\%)\end{array}$ & $\begin{array}{c}\text { Resistance } \\
\text { score }\end{array}$ \\
\hline Block & 1 & $\sigma^{2}+100 \sigma^{2}$ & $0.112^{*}$ & 0.640 \\
Cross & 49 & $\sigma^{2}+2 \sigma^{2}$ & $0.069^{* *}$ & $0.745^{* *}$ \\
Within cross & 49 & $\sigma^{2}$ & 0.027 & 0.183 \\
$\sigma^{2}$ & & & 0.021 & 0.282 \\
${ }^{2}$ & & & 0.61 & 0.88 \\
SE & & & 0.21 & 0.24 \\
\hline
\end{tabular}

Table 4. Regression analyses results and offspring-parent regression heritability estimates for variables scored to describe resistance to Verticillium dahliae in the California strawberry population.

\begin{tabular}{lccc}
\hline \hline Source & df & $\begin{array}{c}\text { Stunted or dead } \\
(\%)\end{array}$ & $\begin{array}{c}\text { Resistance } \\
\text { score }\end{array}$ \\
\hline Regression & 1 & $0.319^{* *}$ & $3.418^{* *}$ \\
Residual & 39 & 0.021 & 0.224 \\
$\sigma_{\mathrm{op}}$ & & 0.018 & 0.178 \\
$\mathrm{~h}^{2}$ & & 0.44 & 0.47 \\
$\mathrm{SE}$ & & 0.11 & 0.12 \\
\hline
\end{tabular}


Table 5. Genetic variance estimates for variables scored to describe resistance to Verticillium dahliae using three methods of comparing genotypes and seedlings.

\begin{tabular}{|c|c|c|c|c|}
\hline \multirow[b]{2}{*}{$\begin{array}{l}\text { Comparison } \\
\text { method }\end{array}$} & \multirow[b]{2}{*}{ Parameter } & \multirow[b]{2}{*}{ Formula } & \multicolumn{2}{|c|}{ Trait } \\
\hline & & & $\begin{array}{c}\text { Stunted or dead } \\
(\%)\end{array}$ & $\begin{array}{c}\text { Resistance } \\
\text { score }\end{array}$ \\
\hline \multirow[t]{2}{*}{ Genotype/OP ${ }^{z}$ covariance } & $\sigma_{\mathrm{a}}^{2}$ & $2 \sigma_{\mathrm{OP}}$ & 0.036 & 0.360 \\
\hline & $\sigma_{\mathrm{d}}^{2}$ & $\sigma_{\mathrm{g}}^{2}-2 \sigma_{\mathrm{OP}}$ & 0.026 & 0.264 \\
\hline \multirow[t]{2}{*}{ Genotype/cross } & $\sigma_{a}^{2}$ & $4 \sigma_{c}^{2}-\sigma_{g}^{2}$ & 0.022 & 0.504 \\
\hline & $\sigma_{\mathrm{d}}^{2}$ & $2\left(\sigma_{g}^{2}-2 \sigma_{c}^{2}\right)$ & 0.040 & 0.120 \\
\hline \multirow[t]{2}{*}{ Cross/OP covariance } & $\sigma_{a}^{2}$ & $2 \sigma_{\mathrm{OP}}$ & 0.036 & 0.360 \\
\hline & $\sigma_{d}^{2}$ & $2\left(\sigma_{c}^{2}-\sigma_{O P}\right)$ & 0.012 & 0.416 \\
\hline
\end{tabular}

${ }^{\mathrm{z}} \mathrm{OP}=$ offspring-parent.

somewhat variable $\left(\sigma_{\mathrm{a}}^{2} / \sigma^{2}{ }_{\mathrm{d}}=0.87\right.$ to 4.20$)$; these heterogeneous estimates might result either from violating some of the assumptions of the genetic model, such as the presence of some epistasis, or from the systematic differences in the experiments discussed previously. Regardless, substantial additive genetic variance was detected in all comparisons; substantial progress can be expected in improving the overall population for resistance and, thus, for improving the frequency of resistant types.

All of the genotypes tested in our study, including the resistant checks, showed some disease symptoms when subjected to the high inoculum rates administered. The observation of disease symptoms in the non-California genotypes may trace in part to the presence in our inoculum of Verticillium strains other than those present during their earlier evaluation. Variation among Verticillium isolates for virulence has been detected (Bringhurst et al., 1961), although the relative genotypic resistance response was nearly identical across all strains tested. Regardless, the sufficiency of currently available germplasm within the California population must be judged ultimately on the potential for selecting transgressive segregants after recombination. Highly resistant Fragaria $\times$ ananassa or wild relatives have been identified (Maas et al., 1989), but their utility in a breeding program depends on whether they carry novel alleles for resistance and the extent to which inoculum thresholds likely to be encountered in commercial production systems will require resistance greater than that expressed in our trials. There is always a substantial cost to using germplasm from either wild relatives or nonadapted sources, and infusion of such germplasm should be used only when absolutely needed (Simmonds 1976).

\section{Literature Cited}

Bringhurst, R.S., P.E. Hansche, and V. Voth. 1968. Inheritance of Verticillium wilt resistance and the correlation of resistance with performance traits. Proc. Amer. Soc. Hort. Sci. 92:369-375.

Bringhurst, R.S., S. Wilhelm, and V. Voth. 1961. Pathogen variability and breeding Verticillium wilt resistant strawberries. Phytopathology 51:786-794.

Bringhurst, R.S., S. Wilhelm, and V. Voth. 1966. Verticillium wilt resistance in natural populations of Fragaria chiloensis in California. Phytopathology 56:219-222.

Comstock, R.E. and H.F. Robinson. 1948. The components of genetic variation in populations of bi-parental progenies and their use in estimating the average degree of dominance. Biometrics 4:254-256.

Falconer, D.S. 1981. Introduction to quantitative genetics. Longman Press, New York.

Maas, J.L. 1984. Compendium of Strawberry Diseases. Amer. Phytopathol. Soc. Press, St. Paul, Minn.

Maas, J.L., G.J. Galletta, and A.D. Draper. 1989. Resistance in strawberry to races of Phytophthora fragaria and to isolates of Verticillium from North America. Acta Hort. 265:521-526.

Nelson, M.D., W.D. Gubler, and D.V. Shaw. 1995. Inheritance of powdery mildew resistance in greenhouse-grown versus field-grown California strawberry progenies. J. Amer. Phytopathol. Soc. 85(4):421-424.

Paulus, A.L. 1990. Fungal diseases of strawberry. HortScience 25:885-889.

SAS Institute. 1988. SAS/STAT users guide. release 6.03. SAS Institute, Cary, N.C. Shaw, D.V., R.S. Bringhurst, and V. Voth. 1989. Genetic parameters estimated for an advanced-cycle strawberry breeding population at two locations. J. Amer. Soc. Hort. Sci. 114:823-827.

Shaw, D.V. 1993. Genetic correlations between vegetative growth traits and productivity at different within- season intervals for strawberries (Fragaria $\times$ ananassa). Theor. Appl. Genet. 85:1001-1009.

Simmonds, N.W. 1986. Plant breeding: the state of the art. Genet. Eng. Plants Symp., Univ. of California, Davis.

Thomas, H.E. 1932. Verticillium wilt of strawberries. Univ. Calif. Agr. Expt. Sta. Bul. 530

Voth, V. and R.S. Bringhurst. 1990. Cultural and physiological manipulation of strawberries. HortScience 25:889-892.

Welch, N. 1989. Strawberry production in California. Univ. Calif. Coop. Ext. Lflt. 2959.

Wilhelm, S. and E.C. Koch. 1956. Verticillium wilt controlled. Calif. Agr. 10:3-14. Wilhelm, S. and A.O. Paulus. 1980. How soil fumigation benefits the California strawberry industry. Plant Dis. 64:264-270.

Wilhelm, S., R.C. Storkan, and J.E. Sagen. 1961. Verticillium wilt of strawberry controlled by fumigation of soil with chloropicrin and chloropicrin-methyl bromide mixtures. Phytopathology 51:744-748. 